\title{
The Design of the Best Penetration Maneuvers Parameter of Variable Trajectory Maneuvers of Target Missiles
}

\author{
Kaibo Bi, Yifei Zhang and Xianhui Sui \\ Dept. of Missile, Dalian Naval Academy, Liaoning Dalian,china,116018
}

\begin{abstract}
To solve the design problem of variable trajectory maneuver of target missiles, an integrated design model of variable trajectory maneuver is presented. By closely cooperating between the control signals of displacements and the control signals of overloads, this integrated design model can make target missiles to realize the maneuver of jump trajectory, the maneuver of snake-wriggling trajectory, the maneuver of pendulum trajectory, the maneuver of spiral trajectory, and so on. On this basis, the penetration effect models of variable trajectory maneuvers of target missiles are researched. The design methods of the best penetration maneuvers parameter is put forward. According to the analysis, target missiles should enlarge maneuver amplitude $A_{y}$ and $A_{z}$ as much as possible within the overload limit. At the same time, we select the optimal parameter $k_{y}^{o p t}$ and $k_{z}^{o p t}$ according to various maneuver modes. According to these parameters, variable trajectory maneuver of target missiles can realize the best penetration effect, and promote train effect of air defense missile.
\end{abstract}

Keywords-target missile; variable trajectory maneuvers; penetration effect formatting

\section{INTRODUCTION}

Currently, many countries with developed military force have already equipped with the terminal maneuver anti-ship missiles[1-4]. For example, American "Harpoon Missile" is a kind of anti-ship missiles with terminal jump maneuver. Russian "Moskit Missile" is equipped with terminal snakewriggling maneuver[5]. Besides, there are some ballistic missiles possess maneuvers of spiral trajectory or maneuvers of pendulum trajectory when they enter into aerosphere. These maneuvers of trajectory have greatly improved the penetration ability of these missiles. In a sense, we can assume that as long as the missiles undertake maneuver flights at certain intension, the penetration ability can be enhanced. Therefore, in military training, resisting target missiles with terminal maneuver trajectory ability plays an important role in testing the real combat capability of aerial defense[6-9].

Compared with attitude control system, overload control system has faster tracing speed and higher accuracy, so overload control system can facilitate the whole process of controlling terminal maneuver trajectory. This study delves into four kinds of integrated design models such as the maneuver of jump trajectory, the maneuver of snake-wriggling trajectory, the maneuver of pendulum trajectory, the maneuver of spiral trajectory, on the base of which, we establish penetration effect models of variable trajectory maneuvers of target Missiles and proposed various design methods of the best penetration maneuvers parameter.

\section{GENERAL DESIGN OF FORMS OF MANEUVER TRAJECTORY TARgET MissiLES}

According to target missiles as the maneuver of jump trajectory, the maneuver of snake-wriggling trajectory, the maneuver of pendulum trajectory, the maneuver of spiral trajectory, we can design their displacement order signal and overload order signal respectively. Generally, individual order signal cannot be used in common, which is not beneficial for engineering application. In order to facilitate the designing process of variable trajectory maneuvers of target missiles, this study proposes an integrated design model of variable trajectory.

Trajectory maneuvers of target missiles is the trajectory of missile centroid relative to ground coordinate Oxyz. Set the longitudinal displacement $x$ of the target projectile as the independent variable, height command signal and the course signal are all $x$ functions. We can get the general design form of variable trajectory of maneuver through summing up height command signals and the course signals of the maneuver of jump trajectory, the maneuver of snake-wriggling trajectory, the maneuver of pendulum trajectory and the maneuver of spiral trajectory:

$$
\left\{\begin{array}{l}
x=x \\
y=y_{1}-\operatorname{sign}\left(\sin \left(\xi_{0}\right)\right) l_{y}+l_{y} \sin \left(\xi_{0}+k_{y} \pi \frac{x-x_{1}}{x_{2}-x_{1}}\right) \\
z=z_{1}-\operatorname{sign}\left(\cos \left(\xi_{0}\right)\right) l_{z}+l_{z} \cos \left(\xi_{0}+k_{z} \pi \frac{x-x_{1}}{x_{2}-x_{1}}\right)
\end{array}\right.
$$

In this formula, $l_{y}, l_{z}, k_{y}, k_{z}$ and $\xi_{0}$ are design parameters of variable trajectory. 
Through calculating the coefficients of the general form of variable trajectory maneuver target missiles (1) for two times, we can get the projection quantum of maneuver acceleration on the three axes in the ground coordinate:

$$
\left\{\begin{array}{l}
a_{x}=0 \\
a_{y}=l_{y} k_{y} \pi \frac{\ddot{x}}{x_{2}-x_{1}} \cos \left(\xi_{0}+k_{y} \pi \frac{x-x_{1}}{x_{2}-x_{1}}\right)-l_{y}\left(k_{y} \pi \frac{\dot{x}}{x_{2}-x_{1}}\right)^{2} \sin \left(\xi_{0}+k_{y} \pi \frac{x-x_{1}}{x_{2}-x_{1}}\right) \\
a_{z}=-l_{z} k_{z} \pi \frac{\ddot{x}}{x_{2}-x_{1}} \sin \left(\xi_{0}+k_{z} \pi \frac{x-x_{1}}{x_{2}-x_{1}}\right)-l_{z}\left(k_{z} \pi \frac{\dot{x}}{x_{2}-x_{1}}\right)^{2} \cos \left(\xi_{0}+k_{z} \pi \frac{x-x_{1}}{x_{2}-x_{1}}\right)
\end{array}\right.
$$

In order to realize the variable trajectory maneuver of target missiles, the cooperation and coordination of overload command signal and displacement command signal is a necessity and the selection of design parameters should comply with the real situation.

\section{Analysis of Penetration Model}

In order to enhance anti-ship missiles' aerial defense ability and combat capability under complicated conditions, it is necessary to analyze the penetration effect of variable trajectory maneuver of target missiles deeply. This section takes the effort miss of interception as an index to evaluate penetration effect of target missiles. We employ the interception-penetration system which has been showed in figure1 as a model in this study.

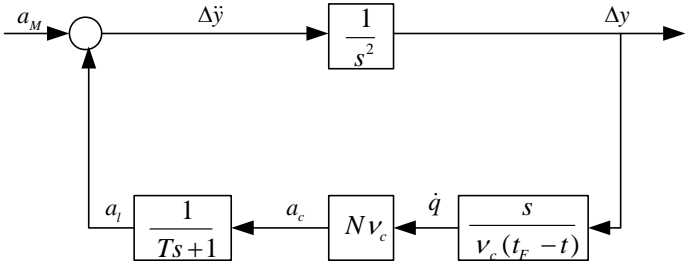

FIGURE I. SIMPLIFIED MODEL OF INTERCEPTION-PENETRATION SYSTEM

In figure I, $a_{M}=f(t) \cdot 1(t)$ is the acceleration of target missile maneuver; $\Delta y$ is the deviation of interception missile against the height of target missile's position; $\frac{S}{v_{c}\left(t_{F}-t\right)}$ is simplified transfer function of the; $v_{c}$ the adjacent speed between target missile and interception missile; $N v_{c}$ is the proportional guidance law for intercepting missile; $\dot{q}$ is the line of sight rate of the interception missile; $\frac{1}{T s+1}$ is the simplified transfer function of interception missile in the controlling process; $n_{c}$ is the command acceleration of the interception missile; $n_{l}$ is the maneuver acceleration of the interception missile.

It is based on conjugated system method, Figure 1conjugated system is shown in Figure II.

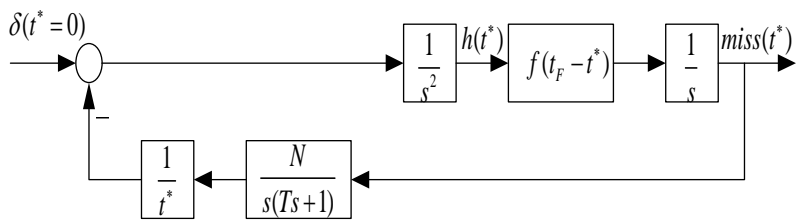

FIGURE II. THE CONJUGATED SYSTEM OF SIMPLIFIED MODEL OF INTERCEPTION-PENETRATION MODEL

In Figure 2, $t^{*}=t_{F}-t$ is time variable of conjugated system; $\delta\left(t^{*}\right)$ is impulse input; $h\left(t^{*}\right)$ is the impulse response of conjugated system.; $f\left(t_{F}-t^{*}\right)$ is the function of maneuver acceleration of target missiles; miss $\left(t^{*}\right)$ is the convolution of the product of $h\left(t^{*}\right)$ and $f\left(t_{F}-t^{*}\right)$, that is $\operatorname{miss}\left(t^{*}\right)=\int_{0}^{t^{*}} f\left(t_{F}-\tau\right) h(\tau) d \tau, \operatorname{miss}\left(t^{*}\right)$ is the terminal maneuver of the target missile at the time $t=t_{F}-t^{*}$, lasting for $t^{*}$ and evocate miss effort at time. Accordingly, there exists such kind of relationship in complex domain:

$$
M(s)=F(s) H(s)
$$

In this formula, $M(s), F(s), H(s)$ are the laplace transformation of $\operatorname{miss}\left(t^{*}\right), f\left(t_{F}-t^{*}\right)$, and $h\left(t^{*}\right)$.

It can be deduced from Figure II:

$$
H(s)=\frac{1}{s^{2}}\left(\frac{T s}{T s+1}\right)^{N}
$$

In this formula, $N$ is the effective navigation ratio of the interception missile. For first-order guidance system, transfer function $H(s)$ shows the transitive relation from the maneuvering acceleration of target missile to the miss efforts of interception missile.

Although the analyzing method only suits for the maneuver of target missiles in a flat, we can extend this method to analyzing the maneuver of three dimensions (the maneuver of pendulum trajectory, the maneuver of spiral trajectory), mainly through dividing the maneuver of three dimensions into longitudinal flat maneuver and course plat maneuver. According to the projection quantum of maneuver acceleration on three axes in ground coordinate, as shown in (2), assuming that the target missile undertakes uniform motion on axis $\mathrm{X}$, 
that is $\dot{x} \neq 0, \ddot{x}=0$, for the convenience of research, we record the quantum of target missiles' maneuver acceleration as:

$$
\left\{\begin{array}{l}
a_{x}=0 \\
a_{y}=-A_{y} \sin \left(\xi_{0}+\omega_{y} t^{*}\right) \\
a_{z}=-A_{z} \cos \left(\xi_{0}+\omega_{z} t^{*}\right)
\end{array}\right.
$$

In this formula, $A_{y}=l_{y}\left(k_{y} \pi \frac{\dot{x}}{x_{2}-x_{1}}\right)^{2}$, $\omega_{y}=\frac{k_{y} \pi V_{x}}{x_{2}-x_{1}} \quad, \quad A_{z}=l_{z}\left(k_{z} \pi \frac{\dot{x}}{x_{2}-x_{1}}\right)^{2} \quad, \quad \omega_{z}=\frac{k_{z} \pi V_{x}}{x_{2}-x_{1}}$, $x-x_{1}=V_{x} t^{*}$.

Therefore, when we take the longitudinal flat maneuver of the target missile into account, $f\left(t_{F}-t^{*}\right)=-A_{y} \sin \left(\xi_{0}+\omega_{y} t^{*}\right)$; when we take the course of navigation flat maneuver of target missiles into account, $f\left(t_{F}-t^{*}\right)=-A_{z} \cos \left(\xi_{0}+\omega_{z} t^{*}\right)$.

If $f\left(t_{F}-t^{*}\right)=A \sin \left(\xi_{0}+\omega t^{*}\right)$, the miss efforts of the interception missile $\operatorname{miss}\left(t^{*}\right)$ is:

$$
\operatorname{miss}\left(t^{*}\right)=\operatorname{miss}_{1}\left(t^{*}\right)+\operatorname{miss}_{2}\left(t^{*}\right)
$$

In this formula, $\operatorname{miss}_{1}\left(t^{*}\right)$ is temporal miss efforts, miss $_{2}\left(t^{*}\right)$ is stable miss efforts. Because the temporal miss efforts decreases exponentially, it will not affect the final result of miss efforts, so we can just take the stable miss efforts into account.

According to the frequency response characteristic of, we can get the amplitude $K_{H}$ of $H(j \omega)$ and phase angle $\phi_{H}$ :

$$
\begin{aligned}
K_{H} & =\frac{\omega^{N-2} T^{N}}{\left[1+(\omega T)^{2}\right]^{N / 2}} N \geq 3 \\
\phi_{H} & =(N-2) \pi / 2-N \tan ^{-1}(\omega T)
\end{aligned}
$$

Due to the effective navigation ratio of the current air defense missile $N$ can meet the condition that $N \geq 3$, we only analyze the situation with $N \geq 3$.
In view of the influence of $H(s)$, the maneuver acceleration $f\left(t_{F}-t^{*}\right)=A \sin \left(\xi_{0}+\omega t^{*}\right)$ causes the stable miss efforts:

$$
\operatorname{miss}_{1}\left(t^{*}\right)=K_{H} A \sin \left(\xi_{0}+\phi_{H}+\omega t^{*}\right)
$$

When the target missile undertakes longitudinal flat snakewriggling, the stable miss effort is:

$$
\operatorname{miss}_{y}\left(t^{*}\right)=K_{H y} A_{y} \sin \left(\xi_{0}+\phi_{H y}+\omega_{y} t^{*}\right)
$$

In this formula,

$$
K_{H y}=\frac{\omega_{y}^{N-2} T^{N}}{\left[1+\left(\omega_{y} T\right)^{2}\right]^{N / 2}}, \phi_{H y}=\frac{(N-2) \pi}{2}-\frac{N}{\tan \left(\omega_{y} T\right)} .
$$

When the target missile undertakes course flat snakewriggling, the stable miss effort is:

$$
\operatorname{miss}_{\mathrm{z}}\left(t^{*}\right)=K_{\mathrm{Hz}} A_{\mathrm{z}} \sin \left(\xi_{0}+\phi_{\mathrm{Hz}}+\omega_{\mathrm{z}} t^{*}\right)
$$

In this formula:

$$
K_{\mathrm{Hz}}=\frac{\omega_{z}^{N-2} T^{N}}{\left[1+\left(\omega_{z} T\right)^{2}\right]^{N / 2}}, \phi_{\mathrm{Hz}}=\frac{(N-2) \pi}{2}-\frac{N}{\tan \left(\omega_{\mathrm{z}} T\right)} .
$$

When the target missile undertakes course three dimensions the stable miss effort is:

$$
\operatorname{miss}^{\prime}\left(t^{*}\right)=\sqrt{\operatorname{miss}_{y}\left(t^{*}\right)^{2}+\operatorname{miss}_{z}\left(t^{*}\right)^{2}}
$$

Through comparing formula(10), formula(11) and formula(12),we can know that, the miss effort of three dimensions is larger than that of a flat maneuver.

\section{SIMULATION ANALYSIS}

In order to elaborate the effect which variable trajectory maneuver of the target missiles poses on the penetration, we compare the change of interception missiles' miss efforts causes with and without the maneuver of the implementation of maneuver.

The target missile undertakes longitudinal snake-wriggling maneuver, the parameters are: $\xi_{0}=0, k_{y}=6, l_{y}=200$, $k_{z}=0 \quad, \quad l_{z}=0 \quad, V_{x}=320 m / s \quad, \quad x_{1}=5000 m$, $x_{2}=35000 m, T=0.9 s, N=3$,compared with the situation without the implementation of maneuver under the same conditions, the result of the miss efforts of interception missiles are shown in Figure III. 


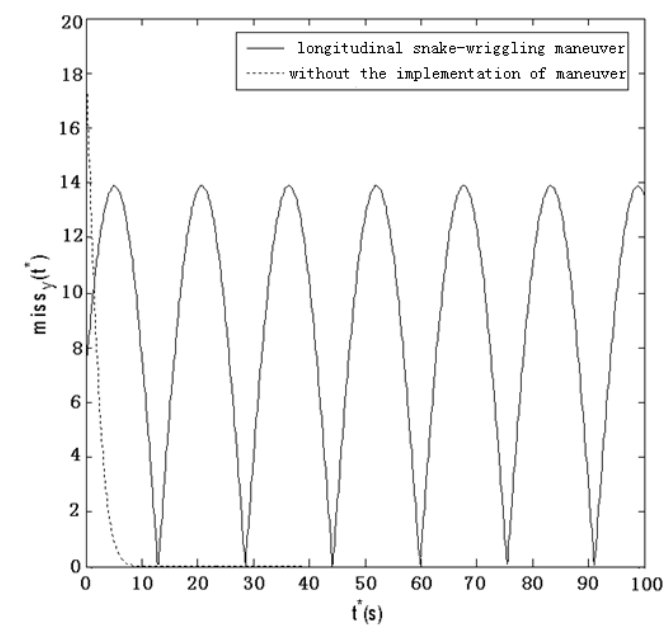

FIGURE III. SIMULATION CURVE OF INTERCEPTION MISSILES' MISS EFFORTS

Comparing the simulation results in figure III we can know that,the real line is the miss effort of the interception missile when it undertakes longitudinal snake-wriggling maneuver; dashed line is the miss effort of the interception missile when it does not undertake longitudinal snake-wriggling maneuver. From the results above, we can know when the target missile does not undertake maneuver flight, the earlier interception is implemented, the smaller the miss effort is, and it is easy to intercept the missiles; however, when the target missile undertakes the snake-wriggling maneuver, the miss effort of interception missiles will show a sinusoidal variation and it increases the uncertainty to intercept the missiles, and at the same time the probability of the penetration will be higher. Therefore, if we design the missile into variable trajectory maneuver target missile, we can further enhance the combat training difficulty, and master the defense technology of resisting missile penetration.

Further more, we can compare the variable trajectory maneuver of three dimensions with the variable trajectory maneuver in the flat, we can trace the discrepancy. If the target missile undertakes the maneuver of pendulum trajectory, we can divide it into longitudinal snake-wriggling maneuver and course snake-wriggling maneuver. Comparing the miss efforts of target missiles of the maneuver of pendulum trajectory, the maneuver of longitudinal snake-wriggling and the maneuver of course snake-wriggling, we can get the simulation results shown in Figure IV.

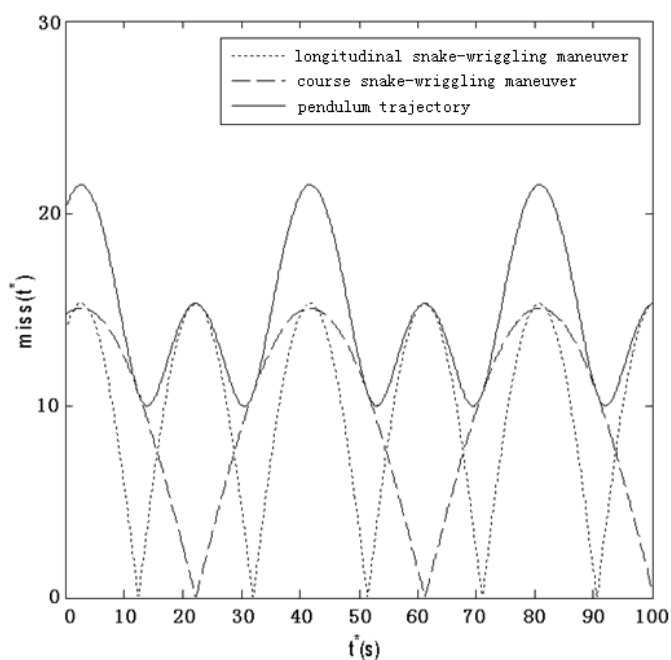

FIGURE IV. SIMULATION CURVE OF INTERCEPTION MISSILES' MISS EFFORTS

Comparing the simulation results in figure IV we can know that, the dashed line is the miss effort of the interception missile when it undertakes longitudinal snake-wriggling maneuver; dotted lineation is the miss effort of the interception missile when it undertakes course snake-wriggling maneuver. The real line is the miss effort of the interception missile when it undertakes pendulum trajectory. Through comparing the results, we can know that when the target missile undertakes longitudinal and course snake-wriggling maneuver, the miss effort of interception missiles shows a fluctuant variation; when the target missile undertakes the maneuver of pendulum trajectory, the maximum of the fluctuant variation of interception missiles' miss effort is much larger than the one of flat snake-wriggling maneuver. There fore, it shows to some extent the probability of undertaking the pendulum maneuver is lower than that of flat snake-wriggling maneuver. It proves the variable trajectory of three dimensions has greater penetration effects than variable trajectory of flat maneuver, and it can further enhance the training performance of interception missiles.

\section{DESIGN OF THE OPTIMAL VARIABLE TRAJECTORY MANEUVER PARAMETER}

Analyzing (10), (11) and (12) we can know that the factors affecting the penetration of target missiles are amplitude of the target missile maneuver $A_{y}$ and $A_{z}$, maneuver frequency $\omega_{y}$ and $\omega_{z}$, interception controlling system time constant $T$ and effective guidance ratio $N$. Because we cannot get accurate value of interception controlling system time constant $T$ and effective guidance ratio $N$, we can analyze the optimal maneuver amplitude and frequency assuming they are under unchangeable condition.

A. Assuming the effective guidance ratio of interception missils $N \geq 3$, taking the longitudinal snake-wriggling 
maneuver as an example, we can get stable miss effort as formula (10).

When we let the stable miss effort of target missiles reach the maximum, which is to refer miss ${ }_{y}\left(t^{*}\right)$ with a max value. Obviously, the larger the maneuver amplitude $A_{y}$ is, the larger the stable miss effort will be. But the value of maneuver amplitude $A_{y}$ is limited by the limitation of the extreme overload, we can enhance the maneuver amplitude under the maximum of target missiles overload. Besides, $\operatorname{miss}_{y}\left(t^{*}\right)$ is the function of maneuver frequency $\omega_{y}$, and $\operatorname{miss}_{y}\left(t^{*}\right)$ has max value. We select optimal $\omega_{y}$ to make sure the maximum $K_{H y}$. We get the first derivative of $\omega_{y}$ against $K_{H y}$.

$$
\frac{\partial K_{H y}}{\partial \omega_{y}}=\frac{(N-2) \omega_{y}^{N-3} T^{N}\left[1+\left(\omega_{y} T\right)^{2}\right]^{\frac{N}{2}}-N \omega_{y}^{N-1} T^{N+2}\left[1+\left(\omega_{y} T\right)^{2}\right]^{\frac{N}{2}-1}}{\left[1+\left(\omega_{y} T\right)^{2}\right]^{N}}
$$

According to the nature of first derivatives, when $\omega_{y}$ satisfies $\frac{\partial K_{H y}}{\partial \omega_{y}}=0$ and $\frac{\partial^{2} K_{H y}}{\partial \omega_{y}^{2}}<0, K_{H y}$ can get maximum. formula

when $\omega_{y}^{\text {opt }}=\frac{\sqrt{2 N-4}}{2 T}, K_{H y}$ reaches maximum $K_{H y}=\frac{T^{2}\left(\frac{N-2}{2}\right)^{\frac{N-2}{2}}}{N^{\frac{N}{2}}}$, and $\operatorname{miss}_{y}\left(t^{*}\right)$ has maximum peak value. According to the formula $\omega_{y}=\frac{k_{y} \pi V_{x}}{x_{2}-x_{1}}$, the speed of target missiles $V_{x}$ is difficult to change and the range of variable trajectory of target missiles $\left[x_{1}, x_{2}\right]$ is settled in advance. Therefore, the designed optimal parameter is $k_{y}^{o p t}=\operatorname{int}\left(\frac{\left(x_{2}-x_{1}\right) \sqrt{2 N-4}}{2 \pi T V_{x}}\right)$, the function $\operatorname{int}(\cdot)$ represents take the complete part of “ . ”, the stable miss effort is maximum. Of course, in order to get the optimal parameter $k_{y}^{o p t}$, we must know the effective guidance ratio $N$ and the control system time constant $T$. There exist some difficulties in real combat. However, the variable trajectory maneuver of target missiles aims at enhance the difficulty of training, and we can estimate the value of $N$ and $T$ of interception missile, and apply it into $\frac{\left(x_{2}-x_{1}\right) \sqrt{2 N-4}}{2 \pi T V_{x}}$, at least we can get less optimal or optimal parameter $k_{y}$, thus realize the less optimal or optimal snake maneuver. Such kind of parameter design method can maximize the stable miss effort of the interception missiles, and enhance the probability of target missiles penetration, and improve the performance of practical training.

As for the course snake-wriggling maneuver of targeted missiles, we can adopt the same analyzing method. The maneuver of jump trajectory of target missile can be regarded as a special example of longitudinal snake-wriggling maneuver, and adopt the same method to analyze it.

B. When the target missiles undertakes the maneuver of pendulum, the stable miss effort is:

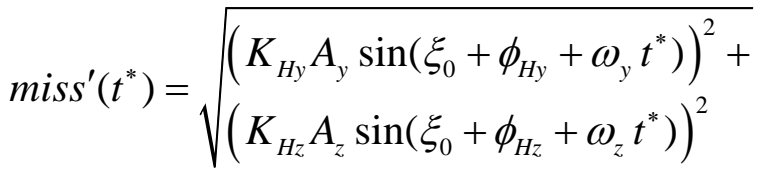

Considering the maneuver of pendulum of target missiles, $k_{y}=2 k_{z}$, according to the definition maneuver frequency $\omega_{y}$ and $\omega_{z}$, we can know $\omega_{y}=2 \omega_{z}$, apply it into (14), we can get

$$
\operatorname{miss}^{\prime}\left(t^{*}\right)=\sqrt{\left(K_{H J} A_{y} \sin \left(\xi_{0}+\phi_{H y}+2 \omega_{z} t^{*}\right)\right)^{2}+\left(K_{H E} A_{z} \cos \left(\xi_{0}+\phi_{H E}+\omega_{z} t^{*}\right)\right)^{2}}
$$

As for formula(15), when maneuver amplitude $A_{y}$, $A_{z}$ reach maximum, stable miss effort $\operatorname{miss}^{\prime}\left(t^{*}\right)$ is higher; so under the max available limit overload condition, we can enhance maneuver amplitude $A_{y}$ and $A_{z}$ as much as possible. Besides, $\operatorname{miss}^{\prime}\left(t^{*}\right)$ is the function of maneuver frequency, and miss $^{\prime}\left(t^{*}\right)$ has max value. We need to select optimal $\omega_{z}$ to realize the maximum of $\operatorname{miss}^{\prime}\left(t^{*}\right)$ in the unit of maneuver period and it is $\operatorname{obj}\left(\omega_{z}\right)=\max \int_{0}^{2 \pi / \omega_{z}} \operatorname{miss} s^{\prime}\left(t^{*}\right) \mathrm{d} t^{*}$. In order to facilitate the study process, we can select the optimal $\omega_{z}$ and make the balance $\operatorname{obj}\left(\omega_{z}\right)=\max \int_{0}^{2 \pi / \omega_{z}}\left(\operatorname{miss}^{\prime}\left(t^{*}\right)\right)^{2} \mathrm{~d} t^{*}$ valid, from this we can get 


$$
\begin{gathered}
\operatorname{obj}\left(\omega_{z}\right)=\max \int_{0}^{2 \pi / \omega_{z}}\left[\begin{array}{l}
\left(K_{H y} A_{y} \sin \left(\xi_{0}+2 \omega_{z} t^{*}+\phi_{H y}\right)\right)^{2}+ \\
\left(K_{H z} A_{z} \cos \left(\xi_{0}+\omega_{z} t^{*}+\phi_{H z}\right)\right)^{2}
\end{array}\right] \mathrm{d} t^{*} \\
=\max \left\{f_{o b j}\left(\omega_{z}\right)\right\}
\end{gathered}
$$

In this formula, $f_{o b j}\left(\omega_{z}\right)=\frac{\pi\left(K_{H y}^{2} A_{y}^{2}+K_{H z}^{2} A_{z}^{2}\right)}{\omega_{z}}$

In order to get the maximum of (16), we calculate the first derivative of $f_{o b j}\left(\omega_{z}\right)$ on $\omega_{z}$

$\frac{\partial f_{o b j}\left(\omega_{z}\right)}{\partial \omega_{z}}=\frac{2 \pi \omega_{z} A_{y}^{2} K_{H y} \frac{\partial K_{H y}}{\partial \omega_{z}}+2 \pi \omega_{z} A_{z}^{2} K_{H z} \frac{\partial K_{H z}}{\partial \omega_{z}}-\pi A_{y}^{2} K_{H y}^{2}-\pi A_{z}^{2} K_{H z}^{2}}{\omega_{z}^{2}}$

In this formula,

$\frac{\partial K_{H y}}{\partial \omega_{z}}=\frac{2^{N-2}(N-2) \omega_{z}^{N-3} T^{N}\left[1+\left(2 \omega_{z} T\right)^{2}\right]^{\frac{N}{2}}-2^{N-1} N \omega_{z}^{N-1} T^{N+2}\left[1+\left(2 \omega_{z} T\right)^{2}\right]^{\frac{N}{2}-1}}{\left[1+\left(2 \omega_{z} T\right)^{2}\right]^{N}}$

$\frac{\partial K_{H z}}{\partial \omega_{z}}=\frac{(N-2) \omega_{z}^{N-3} T^{N}\left[1+\left(\omega_{z} T\right)^{2}\right]^{\frac{N}{2}}-N \omega_{z}^{N-1} T^{N+2}\left[1+\left(\omega_{z} T\right)^{2}\right]^{\frac{N}{2}-1}}{\left[1+\left(\omega_{z} T\right)^{2}\right]^{N}}$

According to the nature of first derivative, when $\omega_{z}$ satisfies $\frac{\partial f_{o b j}\left(\omega_{z}\right)}{\partial \omega_{z}}=0$ and $\frac{\partial^{2} f_{o b j}\left(\omega_{z}\right)}{\partial \omega_{z}^{2}}<0, f_{o b j}\left(\omega_{z}\right)$ can get the maximum. Although it is difficult to get the analysis formula about $\omega_{z}$, from direct calculating formula (17), we can employ graphical construction method, using graphical diagram to represent the relation between $\partial f_{o b j}\left(\omega_{z}\right) / \partial \omega_{z}$ and $\omega_{z}$. Supposing $N=3$ and $T=0.9 s$, the maximum amplitude of target missile $A_{y}=7.0 \mathrm{~g}, A_{z}=5.6 \mathrm{~g}$, the relation between function $\partial f_{o b j}\left(\omega_{z}\right) / \partial \omega_{z}$ and $\omega_{z}$ is shown in Figure V.

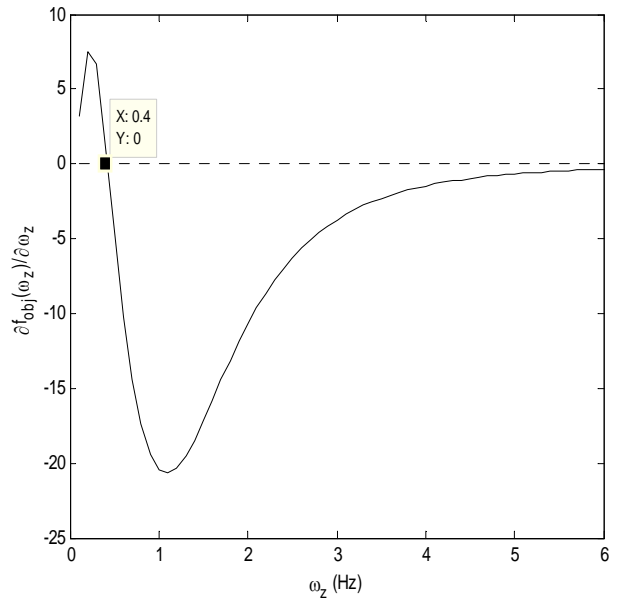

FIGURE V. RELATION BETWEEN FUCTION

$$
\partial f_{o b j}\left(\omega_{z}\right) / \partial \omega_{z} \text { AND } \omega_{z}
$$

We can find the optimal frequency $\omega_{y}^{o p t}=2 \omega_{z}^{o p t}$ and $\omega_{z}^{o p t}$ of pendulum maneuver accurately through graphic construction method. Thus we can get the optimal parameter $k_{y}^{o p t}=\operatorname{int}\left(\frac{\omega_{y}^{o p t}\left(x_{2}-x_{1}\right)}{\pi V_{x}}\right) \quad$ and $k_{z}^{o p t}=\operatorname{int}\left(\frac{\omega_{z}^{o p t}\left(x_{2}-x_{1}\right)}{\pi V_{x}}\right)$.

C. When the target missile takes spiral maneuver, $k_{y}=k_{z}$ and $l_{y}=l_{z}$, according to maneuver amplitude $A_{y}$, $A_{z}$ and the definition of maneuver frequency $\omega_{y}$ and $\omega_{z}$, we can know $K_{H y}=K_{H z}, A_{y}=A_{z}$ and $\omega_{y}=\omega_{z}$, therefore, the miss effort of target missile of spiral maneuver is:

$$
\operatorname{miss}^{\prime}\left(t^{*}\right)=\sqrt{2\left(K_{H y} A_{y} \sin \left(\xi_{0}+\phi_{H y}+\omega_{y} t^{*}\right)\right)^{2}}
$$

According to (20), the higher the maneuver amplitude $A_{y}$ is, the higher miss' $\left(t^{*}\right)$ is; therefore, under the maximum of target missile overland condition, we can enhance maneuver amplitude as much as possible. Besides, $\operatorname{miss}^{\prime}\left(t^{*}\right)$ is the function of maneuver frequency $\omega_{y}, \operatorname{miss}^{\prime}\left(t^{*}\right)$ has the maximum value, and we select the optimal $\omega_{y}$ which can make $\operatorname{miss}^{\prime}\left(t^{*}\right)$ get the maximum value in the unit of maneuver period, that is $\operatorname{obj}\left(\omega_{y}\right)=\max \int_{0}^{2 \pi / \omega_{y}} \operatorname{miss}\left(t^{*}\right) \mathrm{d} t^{*}$. In order to facilitate the study process, we can get the optimal $\omega_{y}$ which 
can make $\operatorname{obj}\left(\omega_{y}\right)=\max \int_{0}^{2 \pi / \omega_{y}}\left(\operatorname{miss}^{\prime}\left(t^{*}\right)\right)^{2} \mathrm{~d} t^{*}$, thereby, we can get

$$
\begin{aligned}
\operatorname{obj}\left(\omega_{y}\right) & =\max \int_{0}^{2 \pi / \omega_{y}} 2\left(K_{H y} A_{y} \sin \left(\xi_{0}+\phi_{H y}+\omega_{y} t^{*}\right)\right)^{2} \mathrm{~d} t^{*} \\
& =\max \left\{f_{o b j}\left(\omega_{y}\right)\right\}
\end{aligned}
$$

In this formula, $f_{o b j}\left(\omega_{y}\right)=\frac{2 \pi K_{H y}^{2} A_{y}^{2}}{\omega_{y}}$.

In order to get the maximum of formula (21), we calculate first derivative of $f_{o b j}\left(\omega_{y}\right)$ on $\omega_{y}$, we can get

$\frac{\partial f_{o b j}\left(\omega_{y}\right)}{\partial \omega_{y}}=\frac{4 \pi \omega_{y} A_{y}^{2} K_{H y} \frac{\partial K_{H y}}{\partial \omega_{y}}-2 \pi A_{y}^{2} K_{H y}^{2}}{\omega_{y}^{2}}$

When $\omega_{y}$ satisfies $\frac{\partial f_{o b j}\left(\omega_{y}\right)}{\partial \omega_{y}}=0$ and $\frac{\partial^{2} f_{o b j}\left(\omega_{y}\right)}{\partial \omega_{y}^{2}}<0$, $f_{o b j}\left(\omega_{y}\right)$ can get the maximum value. By calculating (22) we can get, when $\omega_{y}^{o p t}=\frac{\sqrt{10 N-25}}{5 T}, f_{o b j}\left(\omega_{y}\right)$ can get maximum value, and then we can make sure the stable miss effort miss' $\left(t^{*}\right)$ get the maximum value in the unit of maneuver period. According to the optimal maneuver frequency $\omega_{y}^{o p t}=\omega_{z}^{o p t}=\frac{\sqrt{10 N-25}}{5 T}$, make sure the optimal parameter is $k_{y}^{o p t}=k_{z}^{o p t}=\operatorname{int}\left(\frac{\left(x_{2}-x_{1}\right) \sqrt{10 N-25}}{5 \pi T V_{x}}\right)$

\section{CONCLUSiON}

Centering on the matter of designing variable trajectory maneuvers of target missiles, this study proposes four kinds of variable trajectories, the maneuver of jump trajectory, the maneuver of snake-wriggling trajectory, the maneuver of pendulum trajectory and the maneuver of spiral trajectory. On basis of this, we undertake various kind of study on variable trajectories of penetration effects. And we undertake the optimal parameter design method of variable trajectories. According to the analysis, target missiles should enlarge maneuver amplitude $A_{y}$ and $A_{z}$ as much as possible within the overload limit when the target missiles undertake variable trajectory maneuver of target missiles. At the same time, we select the optimal parameter $k_{y}^{\text {opt }}$ and $k_{z}^{o p t}$ according to various maneuver modes. Under the influence of these optimal parameters, the variable trajectory maneuver of target missiles can realize the best penetration effects, and further enhance the combat training process of aerial defense missiles. Through the analysis of penetration effects, we can master technologies to defend missile penetration and exert a great value for target missile system and combat usage..

\section{REFERENCES}

[1] Ghawe S N, Ghose D. Pure Proportional Navigation against TimeVarying TargetsManeuvers. IEEE Transactions on Aerospace and Electronic Systems [J].1996;32(5):1336-1347.

[2] Yang C D, Yang C C. Optimal Pure Proportional Navigation for ManeuveringTargets. IEEE Transactions on Aerospace and Electronic Systems [J]. 1997; 33(3):949-957.

[3] WANG Xiao-hu,CHEN Han-fu,ZHANG Min-lian. The model and adaptive estimation algorithm for maneuvering target in the terminal guidance section of the air missile. Journal of Astronautics [J]. 2001; 22(2): 61-70.

[4] ZHANG Tao,PENG Shao-xiong,SONG Gui-bao. Design and optimization of anti-ship cruise missile. Aerodynamic Missile Journal [J].2002,32(7): 16-18.

[5] QU Dong-cai,LIN Tao. Russia 'Moskit' anti-ship missile. Ship borne weapon[J].1997; 5(3):7-11.

[6] JIANG Yu-xian,CUI Jing. The effectiveness of missile swing maneuver strategy. Journal of Beijing university of aeronautics and astronautics [J].2002; 28(2): 133-136.

[7] CUI Jing,JIANG Yu-xian. Effect of dynamic characteristics of missile interception of swing maneuver penetration effect. Journal of Astronautics [J]. 2001,22(5):33-38.

[8] ZHAO Hong-chao,GU Wen-jin,PENG Wen-hua. Design of the control system of anti ship missile based on overload control.Tactical Missile Control Technology [J]. 2004,25(3):1-4

[9] WANG Ting. X type low lateral transfer flight target missile. Journal of projectiles, rockets, missile and guidance [J]. 2007,(5):309-311. 